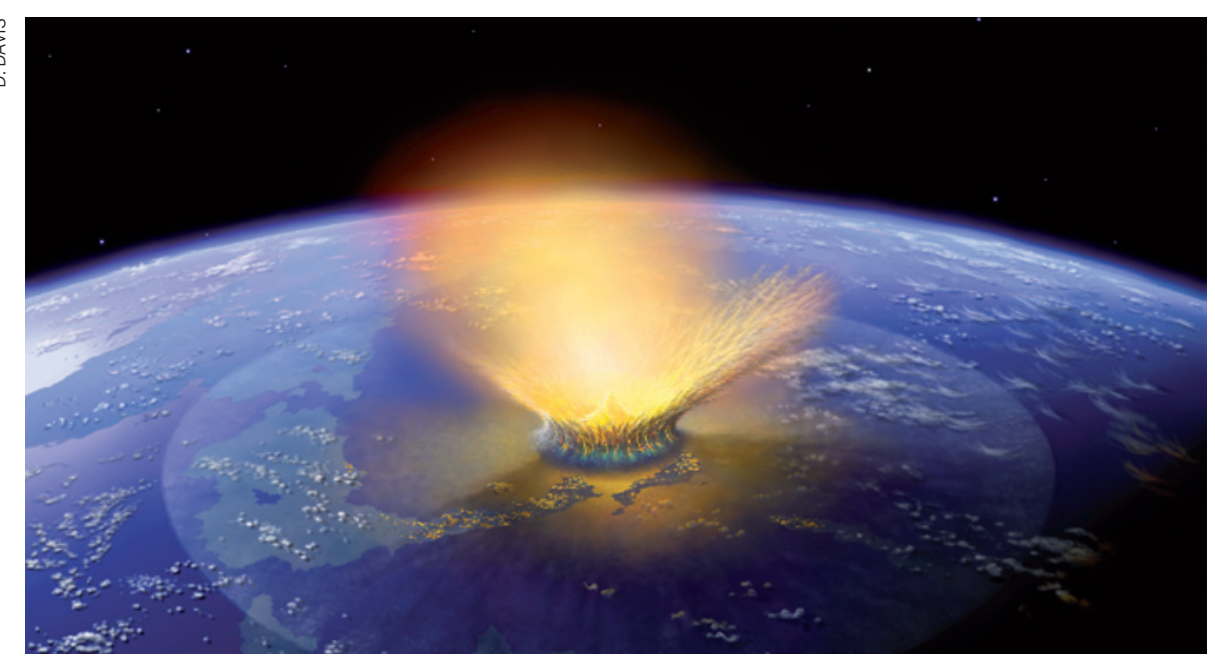

Earth has been struck by as many as 100 impactors as big as those that formed the Moon's giant basins.

PLANETARY SCIENCE

\title{
Ancient asteroids kept on coming
}

\section{Two-billion-year barrage hit Earth when life was beginning.}

\section{BY HELEN THOMPSON}

$\mathrm{T}$ he evidence is in the hollow-eyed face of the man in the Moon: a wave of asteroids showered Earth and the Moon in the distant past. They scarred the lunar surface with vast, circular impact basins and wreaked even greater havoc on this planet, which presented a bigger target. Now, a pair of studies published in Nature ${ }^{1,2}$ suggests that the battering lasted much longer than was thought, spanning nearly the entire first half of Earth's history. The results imply that a prolonged succession of impacts - some of them large enough to vaporize oceans - could have shaped the early evolution of life.

Computer simulations of movements in the early Solar System had suggested that the Late Heavy Bombardment (LHB) that came after the initial accretion of the planets lasted from 4.1 billion to 3.8 billion years ago - a time frame that matches the dates of the lunar basins derived from Moon rocks. The 2005 'Nice model' ${ }^{3}$ showed how, after Uranus and Neptune formed, a shift in the orbits of the outer planets could have destabilized the asteroids in their orbits, putting many on a collision course with the Earth-Moon system. But the model also suggested that the unstable asteroids were quickly depleted, causing the LHB to peter out around the time that the last giant circular impact basin on the Moon was created.

Yet the continuing record of large lunar craters being created after the 3.8-billion-year cut-off, and hints of ancient impacts on Earth, suggested that the story was incomplete. "There had to be something missing," says William Bottke, a planetary dynamicist at the Southwest Research Institute in Boulder, Colorado.

That something, Bottke and his colleagues ${ }^{1}$ now hypothesize, was an extension to the asteroid belt. Currently, asteroids orbit between Mars and Jupiter, with the main belt starting 2.1 astronomical units ( $\mathrm{AU})$ from the Sun ( $1 \mathrm{AU}$ is the distance from the Sun to Earth). Bottke and his colleagues suggest that during the LHB, the inner boundary of the asteroid belt lay just $1.7 \mathrm{AU}$ from the Sun. In computer simulations, the researchers discovered that asteroids dislodged from this extension would

\section{THE LONG TAIL OF BOMBARDMENT}

Simulations hint that the Late Heavy Bombardment (LHB), in which asteroids showered Earth and the Moon, lasted much longer than was thought.

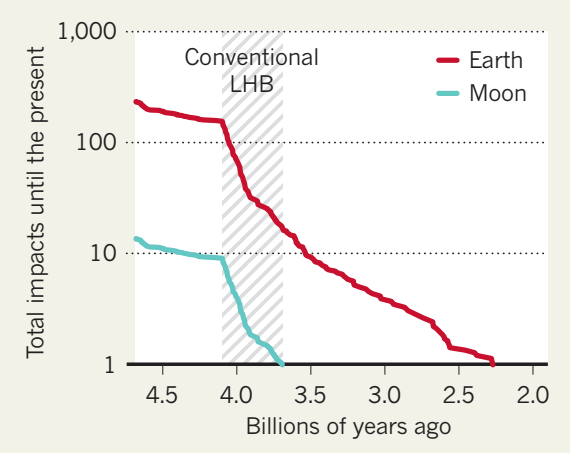

have been ten times more likely to have ended up on Earth-crossing orbits than their mainbelt counterparts. That would have prolonged the LHB on Earth to around 2 billion years ago (see 'The long tail of bombardment').

On Earth, weathering and geological activity have erased any obvious signs of the LHB, but a second study published this week ${ }^{2}$ examined traces of it in the rock record. When an impact splashes molten rock debris into the atmosphere, tiny droplets solidify to form distinctive 'impact spherules'. Impacts as large as the one that may have caused the extinction of the dinosaurs 65 million years ago can scatter spherules around the globe. By looking for similar spherules in layers of rock that formed 2 billion to 3.5 billion years ago, Brandon Johnson and Jay Melosh, geophysicists at Purdue University in West Lafayette, Indiana, found evidence for an extended LHB. They were also able to relate the thickness of each layer and the size of the spherules to the diameter and incoming velocity of the impactor that produced them.

"Our work shows that a lot more big asteroids - meaning dinosaur-killer or larger were hitting Earth well after the current idea of when it ended," says Johnson.

The time frame matches Bottke's model and corresponds to the Archaean aeon. This was a key period in early evolution, which saw the split of the single-celled organisms called archaea from bacteria, and the rise of photosynthesizing cyanobacteria. How life would have responded to a sustained barrage throughout this period is unclear. A giant impact would have come as a severe blow for some forms of early life, but it need not have been all bad news, says Steve Mojzsis, a geologist at the University of Colorado Boulder. That is because the energy deposited by the ongoing impacts could have created hot zones like those found near hydrothermal vents today. "These are great places for microbes," says Mojzsis, who notes that some phylogenetic evidence suggests that the last common ancestors of all present-day life were heat-loving organisms.

The two studies are not in perfect agreement. Johnson and Melosh's work ${ }^{2}$ suggests that the impactors came from near-Earth orbits and hit at around 20 kilometres per second, whereas Bottke's model ${ }^{1}$ has them arriving directly from the asteroid belt at a wider range of speeds. More work will be needed to resolve those differences, says Barbara Cohen, a planetary scientist at NASA's Marshall Space Flight Center in Huntsville, Alabama.

"Both models do a nice job of building on the evidence that we have but, especially on Earth, that evidence is sparse," says Cohen. "The more evidence we find, the better our constraints are going to be." -

1. Bottke, W. F. et al. Nature http://dx.doi. org/10.1038/nature10967 (2012).

2. Johnson, B. C. \& Melosh, J. Nature http://dx.do org/10.1038/nature10982 (2012).

3. Gomes, R., Levison, H. F., Tsiganis, K. \& Morbidelli, A Nature 435, 466-469 (2005). 